Marston Morse and Stewart Scott Cairns

Nagoya Math. J.

Vol. 39 (1970), 167-198

\title{
ELEMENTARY QUOTIENTS OF ABELIAN GROUPS, AND SINGULAR HOMOLOGY ON MANIFOLDS
}

\author{
MARSTON MORSE AND STEWART SCOTT CAIRNS
}

§ 0. Introduction. The topological origin of the problem. Let there be given a compact topological manifold $M_{n}$. If $M_{n}$ admits a "triangulation" it is known that the fundamental invariants, namely the connectivities of $M_{n}$ over fields, the Betti numbers and torsion coefficients over $\boldsymbol{Z}$ of the singular homology groups of $M_{n}$, are finite and calculable. However it is not known that a "triangulaticn" of $M_{n}$ always exists when $n>3$.

Singular homology groups are understood in the sense of Eilenberg [1].

An alternative to triangulation of $M_{n}$. When $M_{n}$ is differentiable, of at least class $C^{2}$, the alternative to the hypothesis of triangulation will be understood to be the existence of a differentiable non-degenerate $(N D)$ function ${ }^{1)} f$ on $M_{n}$. When $M_{n}$ is not known to be so differentiable the alternative will be understood to be the existence in the sense of [2] of a topologically non-degenerate $(T N D)$ function ${ }^{1)} f$ on $M_{n}$.

We shall be concerned with the subsets

$$
f_{c}=\left\{p \in M_{n} \mid f(p) \leq c\right\}
$$

of $M_{n}$ where $c$ is an arbitrary value of $f$ on $M_{n}$ and shall term $f_{c}$ a sublevel set of $M_{n}$.

In a series of papers which make no use of a triangulation of $M_{n}$ we shall show that the fundamental invariants of the singular homology groups I of the sublevel sets $f_{c}$ of $M_{n}$ are uniquely determined by suitable relative numerical invariants associated with the respective critical points on $f_{c}$ of

Received April 21, 1969.

Professor Morse's research was supported in part by U.S. Army Research Office-Durham grant DA-31-124-ARO-D-455.

Professor Cairns' research was supported in part by National Science Foundation grant GP8640.

1) The function $f$ can and will be chosen so as to have different values $a$ at different critical points of $f$. 
a $N D f$. We shall restrict ourselves initially, for simplicity, to the differentiable case.

That the connectivities over fields of the sublevel sets $f_{c}$ are so determined has already been established in [3] in the differentiable case. In this case the invariants associated with a critical point are its index and its type as "linking" or "non-linking". See $\$ 29$ of [3].

The next step is to show how the Betti numbers and torsion coefficients of the sublevel sets $f_{c}$ are determined by suitable relative invariants associated with the critical points. This will be done in [5], beginning with the differentiable case. An abstract of the results in [5] appears as Appendix III of [3].

The objective of the present paper is to solve two problems in abelian group theory, essential in [5]. These group-theoretic problems will be defined at the end of $\S 2$.

The existence of $N D$ functions in the differentiable case has been known and used in singular homology theory since 1927. Cf. $\S 6$ of [3]. TND functions were introduced in [2]. Let $C$ be the class of manifolds $M_{n}$ on which TND functions exist. The class $C$ includes all "combinatorial" manifolds whether differentiable or not. See [7]. If $C^{*}$ is the class of all manifolds $M_{n}$ which admit combinatorial triangulations, it is clear that $C^{*} \subset C$. That this inclusion is proper is believed to be true but has not yet been proved. Cf. [4].

However, the principal reason for the introduction of the new methods was more their directness and relevance than the possibility that they may apply to more general manifolds.

The critical points of $N D$ functions on manifolds, and critical extremals in variational problems must be related to the homology groups of the underlying spaces. It is clearly preferable, when possible, to establish these relations directly without the assumption of triangulability.

It is our ultimate aim to show how the differential or topological invariants, which we associate with the critical points of a $N D$ or TND function $f$, determine the singular homology groups, up to an isomorphism, of all sublevel sets $f_{c}$ on $M_{n}$.

The restriction in this introduction to compact manifolds $M_{n}$ is merely for simplicity.

Introductory remarks on abelian groups $A$. The generation of subgroups of $A$. For $i$ on the range $1, \cdots, r$ let $X_{i}$ be a subset, subgroup, 
or element of $A$. We shall denote by

$$
\left\{X_{1}, X_{2}, \cdots, X_{r}\right\}
$$

the subgroup of $A$ which is the intersection of those subgroups of $A$ which include or contain each $X_{i}$. If $a_{i}$ is an element of $A$ the group

$$
G=\left\{a_{1}, \cdots, a_{r}\right\}
$$

is said to be finitely generated. By virtue of this notation, if $a$ is an element of $A,\{a\}$ denotes the cyclic subgroup generated by $a$.

Definition 0.1. Free subgroups. A subset $\left(u_{1}, \cdots, u_{r}\right)$ of elements $u_{i} \in A$ is termed free, provided a relation $n_{1} u_{1}+\cdots+n_{r} u_{r}=0$ in which $n_{i}$ is an integer is valid if and only if $n_{1}=n_{2}=\cdots=n_{r}=0$. If $\left(u_{1}, \cdots, u_{r}\right)$ is free the subgroup $G=\left\{u_{1}, \cdots, u_{r}\right\}$ is termed free and $\left(u_{1}, \cdots, u_{r}\right)$ a base for $G$. It will be convenient to term the trivial group free, and say that it has an empty base.

Definition 0.2. The torsion subgroup of $A$. The subset of elements of $A$ of finite order form a group, termed the torsion subgroup of $A$.

Definition 0.3. Direct sums. An ensemble $A_{1}, \cdots, A_{n}$ of subgroups of $A$ such that $A=\left\{A_{1}, A_{2}, \cdots, A_{n}\right\}$ will be said to have the direct sum

$$
A=A_{1} \oplus \cdots \oplus A_{n},
$$

provided a relation $x_{1}+x_{2}+\cdots+x_{n}=0, x_{i} \in A_{i}$, is valid only if each $x_{i}=0$. We admit null summands $A_{i}$.

If $\left(u_{1}, \cdots, u_{r}\right)$ is a base for a free subgroup $\mathscr{B}$ of $A$, then

$$
\mathscr{B}=\left\{u_{1}\right\} \oplus \cdots \oplus\left\{u_{r}\right\} .
$$

The order of each cyclic summand is infinite. We admit the case in which $r=0$ and $\mathscr{B}=0$.

Theorem 0.1. A finitely generated abelian group $A$ is a direct sum $A=\mathscr{B} \oplus \mathscr{T}$ of the torsion subgroup $\mathscr{T}$ of $A$, and a free subgroup $\mathscr{B}$ of $A$ termed complementary to $\mathscr{T}$ in $A$ [8], p. 151.

A group complementary to the torsion subgroup $\mathscr{T}$ of $A$ is uniquely determined if and only if $\mathscr{T}=0$ or $\mathscr{T}=A$. The most general group complementary to $\mathscr{T}$ has the form

$$
\left\{v_{1}+t_{1}\right\} \oplus \cdots \oplus\left\{v_{r}+t_{.}\right\}
$$


where $\left(v_{1}, \cdots, v_{r}\right)$ is an arbitrary base of $\mathscr{B}$ and $t_{1}, \cdots, t_{r}$ arbitrary elements in $\mathscr{T}$.

Definition 0. 4. Betti subgroups of $A$. When $A$ is ${ }^{2)} F G$ we shall call a subgroup $\mathscr{B}$ complementary to the torsion subgroup $\mathscr{T}$ of $A$ a Betti subgroup of $A$, and its dimension the Betti number of $A$.

We shall be concerned with abelian groups $A$ which are $F G$.

§1. Sylow groups, elementary divisors, and torsion coefficients. We shall recall certain terms and theorems associated with decomposing a finite abelian group $\mathscr{T}$ into a direct sum of cyclic subgroups.

Definition 1. 1. Prime powers. An integer $q>1$ admits a factoring

$$
q=p_{1}^{e_{1}} \cdots p_{r}^{e_{r}}, \quad(r>0)
$$

unique except for the order of the factors, in which the $p_{i}$ 's are distinct positive primes and the $e_{i}$ 's positive integers. Such a factoring will be called a reduced prime power factoring of $q$. Hereafter primes are supposed positive.

Definition 1.2. $p$-Primary subgroups of $\mathscr{T}^{-}$. A subgroup $g$ of $\mathscr{T}$ whose order is a power of a prime $p$ is called a $p$-primary subgroup of $\mathscr{T}$. A maximal $p$-primary subgroup of $\mathscr{T}$ is the union, for a given prime $p$, of all $p$-primary subgroups of $\mathscr{T}$. It is called a Sylow $p$-subgroup of $\mathscr{T}$ and will be denoted by $\mathscr{S}_{p}$.

Two classical theorems follow.

Theorem 1.1. A finite abelian group $\mathscr{T}$, with an order $q>1$ given by (1.1), is the direct sum

$$
\mathscr{T}=\mathscr{S}_{p_{1}} \oplus \cdots \oplus \mathscr{S}_{p_{r}}
$$

of the nontrivial Sylow subgroups of $\mathscr{T}$ [9], p. 137.

Theorem 1.2. The "cyclic primary decomposition" theorem." A finite nontrivial abelian group $\mathscr{T}$ is the direct sum

$$
\mathscr{T}=g_{1} \oplus \cdots \oplus g_{m},
$$

2) $F G$ abbreviates finitely generated.

3) CPD shall abbreviate "cyclic primary decomposition". 
of nontrivial primary cyclic subgroups $g_{i}$ of $\mathscr{T}$ uniquely determined by $\mathscr{T}$ up to isomorphisms and order of writing of the summands. [10], pp. 60, 65.

The numerical values of the orders of the $g_{i}$ 's are prime powers. These prime powers form a list

$$
p_{1}^{e_{1}}, \cdots, p_{m}^{e_{m}} \quad\left(e_{i}>0\right)
$$

of prime powers, not necessarily distinct. We shall say that the groups $g_{i}$ of (1.3) and the prime powers (1.4) are normally ordered if

$$
p_{1} \geq p_{2} \geq \cdots \geq p_{m}
$$

and if when $p_{i}=p_{i+1}$, then $e_{i} \geq e_{i+1}$.

According to Theorem 1.2, the prime powers (1.4), if normally ordered, are uniquely determined by $\mathscr{T}$.

Definition 1.3. Elementary divisors of $\mathscr{T}$. The prime powers in (1.4) are called the elementary divisors of $\mathscr{T}$, and are regarded as algebraically distinct if they have distinct indices $i$ in (1.4). By the multiplicity of an elementary divisor $p_{i}^{e_{i}}$ of $\mathscr{T}$ is meant the number of algebraically distinct elementary divisors of $\mathscr{T}$ with the same numerical value as $p_{i}^{e_{i}}$. Theorem 1.2 implies that the elementary divisors of $\mathscr{T}$, together with their multiplicities, are uniquely determined by $\mathscr{T}$.

Definition 1.4. Torsion coefficients of $\mathscr{T}$. It is a classical theorem that a finite non-trivial trivial abelian group $\mathscr{T}$ is a direct sum of a finite set of cyclic subgroups of $\mathscr{T}$ which have orders

$$
q_{1}, q_{2}, \cdots, q_{\rho}
$$

exceeding 1 , each of which, except $q_{\rho}$, is divisible by its successor. The integers in the sequence (1.6) are uniquely determined by $\mathscr{T}$. They are termed torsion coefficients of $\mathscr{T}$ and are said to be canonically ordered.

The matrix $\Pi_{r \mu}$. Let $r$ be the number of distinct primes $p_{1}, \cdots, p_{r}$ in (1.1). Let these primes be taken in decreasing order. Let $\alpha_{i}$ be the number ${ }^{4}$ of $E D$ 's which are powers of $p_{i}$, and let $\mu$ be the maximum of the numbers $\alpha_{i}$.

The martix $\Pi_{r \mu}$ shall have $r$ rows and $\mu$ columns. The $i$-th row of $\Pi_{r \mu}$ shall consist of the ED's of $\mathscr{T}$. which are powers of $p_{i}$, arranged in

4) $E D$ abbreviates "elementary divisor". 
monotonically decreasing order and followed by enough 1's to make a row of $\mu$-integers. Each element $a_{i j}$ of $\Pi_{r_{\mu}}$ is thus an $E D$ of $\mathscr{T}$ or 1 .

The $E D$ 's of $\mathscr{T}$ and the torsion coefficients of $\mathscr{T}$ uniquely determine each other in accord with the following theorem.

THeOREM 1.3. The torsion coefficients of $\mathscr{T}$ are $\mu$ in number and if "canonically" arranged, are the products of the elements in the respective columns of $\Pi_{r \mu}$. Conversely, the element $a_{i j}$ of $\Pi_{r \mu}$ is the maximal prime power of $p_{i}$ which is a factor of the $j$-th torsion coefficient of $\mathscr{T}$.

This theorem may inferred from [9], p. 147.

Definition 1.5. A "basis" for $A$. Suppose that $\mathscr{T}$ has a $C P D$

$$
\mathscr{T}=\left\{x_{1}\right\} \oplus \cdots \oplus\left\{x_{\rho}\right\} \quad\left(\text { order } x_{i}>1 ; i=1, \cdots, \rho\right)
$$

and that $\mathscr{B}$ is a Betti group of $A$ with a "base" $\left(v_{1}, \cdots, v_{\beta}\right)$. The set of elements

$$
v_{1}, \cdots, v_{\beta} ; x_{1}, \cdots, x_{\rho}
$$

of $A$ is called a basis for $A$. If $\mathscr{B}$ is trivial there are no $v_{i}$ 's, and if $\mathscr{T}$ is trivial no $x_{j}$ 's.

A basis for $A$ is to be distinguished from a base for $\mathscr{B}$ which is free.

A basis for a non-trivial abelian group $A$ is unique only if $A$ is a cyclic group of order 2 .

If $w$ is an arbitrary element in $A$ then

$$
w=\mu_{1} v_{1}+\cdots+\mu_{\beta} v_{\beta}+m_{1} x_{1}+\cdots+m_{\rho} x_{\rho}
$$

where the coefficients $\mu_{i}$ and $m_{j}$ are integers. If the integers $m_{j}$ are restricted to integral values such that

$$
0 \leq m_{j}<\text { order } x_{j}
$$

then the coefficients $m_{j}$, as well as the coefficients $\mu_{i}$, are uniquely determined by $w$ and the choice of the basis (1.8).

Definition 1.6. Prime-simple abelian groups. A power $p^{e}$ of a prime is termed prime-simple if $e=1$. An element $x \in A$ is termed primesimple if $(\operatorname{order} x)=\infty$, or if $(\operatorname{order} x)<\infty$ and each prime power factor of (order $x$ ) is 1 or prime-simple. A subgroup of $A$ (including $A$ ) is termed prime-simple if each of its elements is prime-simple. 
Subgroups of prime-simple groups are prime-simple. The abelian group $A$ is prime-simple if and only if each Sylow subgroup of $A$ is prime-simple. One readily establishes the following theorem.

Theorem 1.4. A necessary and sufficient condition that a finitely generated abelian group be prime-simple is that each elementary divisor of its torsion subgroup be prime-simple.

By the length of a $C P D$ of a finite abelian group we mean the number of nontrivial summands in the $C P D$.

The following theorem gives three fundamental properties of a nontrivial finite cyclic group.

Theorem 1.5 (i). The elementary divisors of a finite cyclic group $G$ are the maximal prime powers in a reduced prime power factoring of the order of $G$. [14], p. 23 .

(ii) Apart from the order of its summands there is but one CPD of a nontrivial finite cyclic group $G$ and this $C P D$ is a Sylow decomposition of $G$.

(iii) A finite nontrivial abelian group $G$ is cyclic if and only if each of its Sylow subgroups has a CPD of unit length.

$\S 2$. The invariants of group quotients $A / \boldsymbol{R}$. The group $A$ is the above finitely generated abelian group $A$, and $\boldsymbol{R}$ a subgroup of $A$. The quotient $A / \boldsymbol{R}$ is $F G$ as we shall presently see. By the invariants of $A / \boldsymbol{R}$ we here mean its Betti numbers, torsion coefficients and elementary divisors.

The problem of finding the invariants of $A / \boldsymbol{R}$ depends formally upon how $A$ and $\boldsymbol{R}$ are given. We suppose that $A$ is an $F G$ abelian group given as a direct sum

$$
A=\mathscr{B} \oplus \mathscr{T}
$$

of the torsion subgroup $\mathscr{T}$ of $A$ and a complementary free subgroup $\mathscr{B}$ of $A$. We suppose that a "base" $\left(u_{1}, \cdots, u_{r}\right)$ of $\mathscr{B}$ is given and generators $x_{1}, \cdots, x_{\rho}$ of the respective summands of a $C P D$ of $\mathscr{T}$, together with the order $n_{i}$ of each $x_{i}$.

In a form more general than any we shall use, there is given a subgroup $\boldsymbol{R}=\left\{z_{1}, \cdots, z_{\alpha}\right\}$ where each generator $z_{i}$ is given as a sum

$$
\left(\nu_{1} u_{1}+\cdots+\nu_{r} u_{r}\right)+\left(m_{1} x_{1}+\cdots+m_{\rho} x_{\rho}\right)
$$

with integral coefficients. The general problem is to find the "invariants" 
of $A / \boldsymbol{R}$ in terms of these data and to do this by a finite well-defined algorithm.

That $A / \boldsymbol{R}$ is $F G$ is a consequence of the fact that the $\boldsymbol{R}$-cosets containing the elements $u_{1}, \cdots, u_{r} ; x_{1}, \cdots, x_{\rho}$ generate $A / \boldsymbol{R}$.

The quotients $A / g$ whose invariants will be studied in this paper readily reduce to quotients which we term correlated and define as follows.

Definition 2.1. Correlated quotients. In such quotients $A$ is given as a direct sum $G_{1} \oplus \cdots \oplus G_{r}$ of subgroups $G_{i}$ and $g$ is a direct sum $g_{1} \oplus \cdots \oplus g_{r}$ of cyclic groups $g_{i}$, where $g_{i}$, is a subgroup, possibly trivial, of $G_{i}$. Under these conditions $A / g$ is termed a correlated quotient.

It is a classically known and easily proved theorem that when $A / g$ is the above correlated quotient then

$$
A / g \approx G / g_{1} \oplus \cdots \oplus G_{r} / g_{r} \quad([10], \text { p. } 57)
$$

where the right member of (2.3) is the "external direct sum" of the quotients $G_{i} / g_{i}$ and the isomorphism is a "natural" isomorphism which we shall now characterize.

External direct sums. The direct sums which we have been considering up to this point have been direct sums of subgroups of a given group. The group quotients on the right of (2.3) are not so given. They can be considered, a priori, as abelian groups $A_{1}, \cdots, A_{r}$ with no elements in common.

If $i$ is on the range $1, \cdots, r$, and $y_{i}$ is an arbitrary element in $A_{i}$, one can form a new abelian group $K$ called the external direct sum of the $A_{i}$ 's. The elements in $K$ are by definition $r$-tuples $\left(y_{1}, \cdots, y_{r}\right)$. These $r$-tuples are summed by adding their respective components $y_{i}$. The inverse of $\left(y_{1}, \cdots, y_{r}\right)$ is $\left(-y_{1}, \cdots,-y_{r}\right)$. One can identify $A_{i}$ with the subgroup of $K$ of elements whose components are 0 except the $i$-th. The $i$-th component shall be an element in $A_{i}$.

An isomorphism (2.3), termed "natural", can be characterized as follows: Let $x_{i}$ be an arbitrary element in $G_{i}$, and $\hat{x}_{i}=x_{i}+g_{i}$ the coset of $g_{i}$ containing $x_{i}$. In the isomorphism (2.3) the coset $\left(x_{1}+x_{2}+\cdots+x_{n}\right)+g$ in $A$ shall go into the $r$-tuple $\left(\hat{x}_{1}, \hat{x}_{2}, \cdots, \hat{x}_{r}\right)$ in the external direct sum of the $r$ quotients $G_{i} / g_{i}$.

Because of the isomorphism (2.3), the problem of finding the "invariants" of "correlated" quotients $\boldsymbol{A} / \mathrm{g}$ reduces to the problem of finding the invariants of quotients of the type which we call elementary and define as follows. 
Definition 2.2 Elementary quotients $A / W$. In such quotients $W$ is a cyclic subgroup $\{w\}$ of $A$.

We shall recall a special type of group quotient, and the mode of reduction of such quotients to quotients of "correlated" type.

Quotients $\boldsymbol{A} / \boldsymbol{R}$ with $\boldsymbol{A}$ free. In such quotients $\boldsymbol{A}$ is a free group given as a direct sum

$$
\boldsymbol{A}=\left\{v_{1}\right\} \oplus \cdots \oplus\left\{v_{o}\right\} \quad(\sigma>0)
$$

of $\sigma$ infinite cyclic groups. $\boldsymbol{R}$ is given as a subgroup of $\boldsymbol{A}$ of the form

$$
\boldsymbol{R}=\left\{z_{1}, \cdots, z_{r}\right\} \quad(r>0)
$$

with generators $z_{i}$ which are integral linear combinations

$$
z_{i}=a_{i j} v_{j} \quad(i=1, \cdots, r)
$$

of the base elements $\left(v_{1}, \cdots, v_{\sigma}\right)$. The terms $a_{i j} v_{j}$ are summed over the range $1,2, \cdots, \sigma$ for $j$.

The diagonal matrix $\left\|d_{i j}\right\|$. Let $\rho>0$ be the rank of the matrix $\left\|a_{i j}\right\|$. It is well known that by a finite sequence of "elementary" operations on the rows and columns of $\left\|a_{i j}\right\|$, the matrix $\left\|a_{i j}\right\|$ can be reduced to an "equivalent" matrix $\left\|d_{i j}\right\|$ with the same number of rows and columns, and with all elements zero, except positive diagonal elements

$$
d_{1}, d_{2}, \cdots, d_{\rho} \quad(\rho \leq \sigma)
$$

of which each $d_{i}$, except the last, divides its successor. The $d_{i}$ 's obtained in this way are termed invariant factors of $\left\|a_{i j}\right\|$ and are uniquely determined by $\left\|a_{i j}\right\|$. [12] p. 308 .

The invariants $D_{j}$. The numbers $d_{i}$ can be determined as follows. For $j=1, \cdots, \rho$ let $D_{j}$ be the absolute value of the $H C F$ of the $j$-rowed subdeterminants of the matrix $\left\|a_{i j}\right\|$. It is known that

$$
d_{1}=D_{1}, d_{2}=\frac{D_{2}}{D_{1}}, \cdots, d_{\rho}=\frac{D_{\rho}}{D_{\rho-1}} \cdot \quad([13] \text { p. 178) }
$$

Torsion coefficients of $\boldsymbol{A} / \boldsymbol{R}$. Let $\boldsymbol{T}$ be the torsion subgroup of $\boldsymbol{A} / \boldsymbol{R}$. We shall show how to determine the torsion coefficients of $\boldsymbol{T}$.

The reduction by elementary operations of the matrix $\left\|a_{i j}\right\|$ to the matrix $\left\|d_{i j}\right\|$ implies the existence of a unimodular transformation of the 
base $\left(v_{1}, \cdots, v_{o}\right)$ into a base $\left(v_{1}^{\prime}, \cdots, v_{\sigma}^{\prime}\right)$, and of a unimodular transformation of the set $\left(z_{1}, \cdots, z_{r}\right)$ into a new set $\left(z_{1}^{\prime}, \cdots, z_{r}^{\prime}\right)$ generating $\boldsymbol{R}$ such that

$$
z_{i}^{\prime}=d_{i j} v_{j}^{\prime} \quad(i=1,2, \cdots, r) .
$$

Because of the diagonal character of $\left\|d_{i j}\right\|, \boldsymbol{A} / \boldsymbol{R}$ is represented by a "correlated" quotient

$$
\frac{\left.\left.\left\{v_{1}^{\prime}\right\} \oplus \cdots \oplus\left\{v_{\rho}^{\prime}\right\} \oplus\left\{v_{\rho+1}^{\prime}\right\} \oplus \cdots \oplus\right\} v_{\sigma}^{\prime}\right\}}{\left\{d_{1} v_{1}^{\prime}\right\} \oplus \cdots \oplus\left\{d_{\rho} v^{\prime}\right\} \oplus\{0\} \oplus \cdots \oplus\{0\}} \quad(\rho \leq \sigma) .
$$

By virtue of the natural isomorphism (2.3) $\boldsymbol{A} / \boldsymbol{R}$ is thus isomorphic to the external direct sum

$$
C\left(d_{1}\right) \oplus \cdots \oplus C\left(d_{\rho}\right) \oplus\left\{v_{\rho+1}\right\} \oplus \cdots \oplus\left\{v_{\sigma}\right\}
$$

where $C\left(d_{i}\right)$ is a cyclic group of order $d_{i}$ and the remaining summands in (2.11) have infinite orders.

This result implies a classical theorem:

Theorem 2.1. When $\boldsymbol{A}$ is free the Betti number of $\boldsymbol{A} / \boldsymbol{R}$ equals $\sigma-\rho$, where $\boldsymbol{\sigma}$ is the dimension of $\boldsymbol{A}$ and $\rho$ the rank of $\left\|a_{i j}\right\|$, while the invariant factors of $\left\|a_{i j}\right\|$ which exceed 1 are the torsion coefficients of $\boldsymbol{A} / \boldsymbol{R}$. [12] p. 308.

We state a useful corollary.

Corollary 2.1. Given an abelian group

$$
\mathscr{T}=C\left(t_{1}\right) \oplus \cdots \oplus C\left(t_{r}\right)
$$

where $t_{1}, \cdots, t_{r}$ are finite integers exceeding 1 , the torsion coefficients of $\mathscr{T}$ are the invariant factors exceeding 1 of the $r$-square diagonal matrix $\left\|t_{i j}\right\|$ with diagonal $t_{1}, \cdots, t_{r}$.

The problems of this paper. In sections 3 and 4 we shall give, in simplified matrix form, an algorithm showing how to compute the invariants of elementary quotients $A / W$ when the invariants of $A$ are given and a generator $w$ of $W$ is given in form (1.9).

In $\S 5$ we shall show how the hypothesis of prime-simplicity of $A$ simplifies the problem of determining the invariants of $A / W$. Necessary and sufficient conditions that $A / W$ be prime-simple when $A$ is prime-simple are given.

5) Summing as to $j$ on the range $1,2, \cdots, \sigma$. 
§3. The elementary quotients $A / W$. The group $A$ is "given" as $A$ was given in $\S 2$. Moreover $W=\{\boldsymbol{w}\}$, where $\boldsymbol{w} \in A$. The giving of $\boldsymbol{w}$ should be such that the data on $\boldsymbol{w}$, together with the invariants of $A$, determine the invariants of $A / W$.

We shall begin by assigning to each $\boldsymbol{w} \in A$ an integer $s \geq 0$ termed the free index of $\boldsymbol{w}$. When $\boldsymbol{w} \notin \mathscr{T}$, this integer $\boldsymbol{s}$ is characterized in Lemma 3.1 .

Notation. In formulating Lemma 3.1 we write $x=y \bmod \mathscr{T}$ whenever $x$ and $y$ are elements in $A$ such that $x-y$ is in $\mathscr{T}$.

Lemma 3.1 (i). Corresponding to a $\boldsymbol{w} \in A$ of infinite order, there exists an integer $s>0$, such that a prescribed Betti group $\mathscr{B}$ of $A$ has a base with first element $u_{B}$ such that

$$
\boldsymbol{w}=\boldsymbol{s} u_{B} \bmod \mathscr{T} .
$$

(ii) If there is given a second Betti group $\mathscr{B}^{\prime}$ of $A$ and a positive integer $\boldsymbol{s}^{\prime}$, such that for some element $u_{B^{\prime}}$ in a base for $\mathscr{B}^{\prime}$,

$$
\boldsymbol{w}=\boldsymbol{s}^{\prime} u_{B^{\prime}} \bmod \mathscr{T},
$$

then $s=s^{\prime}$.

Proof of (i). Let the prescribed Betti subgroup $\mathscr{B}$ of $A$ have a base $\left(v_{1}, \cdots, v_{r}\right), r>0$. For suitable choices of integers $t_{1}, \cdots, t_{r}$, not all zero,

$$
\boldsymbol{w}=t_{1} v_{1}+\cdots+t_{r} v_{r} \bmod \mathscr{T} .
$$

Let $s$ be the $G C D$ of $t_{1}, \cdots, t_{r}$. There then exist integers $k_{1}, \cdots, k_{r}$, whose $G C D$ equals 1 , and which are such that

$$
\boldsymbol{w}=\boldsymbol{s}\left(k_{1} v_{1}+\cdots+k_{r} v_{r}\right) \bmod \mathscr{T} .
$$

It follows from the lemma in [8], p. 145, that

$$
u_{B}=k_{1} v_{1}+\cdots+k_{r} v_{r}
$$

is an element $u_{1}$ in a base $\left(u_{1}, \cdots, u_{r}\right)$ for $\mathscr{B}$, thereby proving (i).

Proof of (ii). Suppose that (3.1) and (3.2) both hold so that

$$
\boldsymbol{s} u_{B}=\boldsymbol{s}^{\prime} u_{B^{\prime}} \bmod \mathscr{T} .
$$

In terms of the base $\left(u_{1}, \cdots, u_{r}\right)$ of $\mathscr{B}$, for suitable integers $\mu_{1}, \cdots, \mu_{r}$, 
(3.6) takes the form

$$
\boldsymbol{s} u_{1}=\boldsymbol{s}^{\prime}\left(\mu_{1} u_{1}+\cdots+\mu_{r} u_{r}\right) \bmod \mathscr{T},
$$

from which we infer that $s=\boldsymbol{s}^{\prime} \mu_{1}$, and hence that $\boldsymbol{s}^{\prime} \leq \boldsymbol{s}$. Similarly $\boldsymbol{s} \leq \boldsymbol{s}^{\prime}$ so that $s=\boldsymbol{s}^{\prime}$.

The proof of (ii) is complete.

To each non-trivial element $\boldsymbol{w} \in A$ we now assign indices $\boldsymbol{s}$ and $\boldsymbol{t}$ as follows.

Definition 3.1. The free index $\boldsymbol{s}$ of $\boldsymbol{w}$. If $\boldsymbol{w} \in \mathscr{T}$ set $\boldsymbol{s}=0$. If $\boldsymbol{w} \oplus \mathscr{T}$, let $\boldsymbol{s}>0$ be the integer affirmed to exist in Lemma 3.1.

By virtue of this definition of $s$

$$
\boldsymbol{w}=\boldsymbol{s} u_{B}+\tau_{B} \quad\left(\tau_{B} \in \mathscr{T}\right)
$$

where $u_{B}$ is either 0 or the first element in a base for $\mathscr{B}$, according as order $\boldsymbol{w}$ is finite or infinite.

Definition 3.2. The torsion index $\boldsymbol{t}$ of $\boldsymbol{w}$. Set

$$
\text { order } \tau_{B}=t_{B}
$$

and

$$
\min _{B} t_{B}=\boldsymbol{t}
$$

where $\mathscr{B}$ ranges over all Betti groups complementary to $\mathscr{T}$ in $A$. We term $\boldsymbol{t}$ the torsion index of $\boldsymbol{w}$. When $\boldsymbol{s}=0, \boldsymbol{t}=t_{B}$ for every choice of $\mathscr{B}$.

There are three theorems corresponding to the three cases $s=0, s=1$ and $s \geq 1$, showing how the invariants of $\boldsymbol{A}$ are related to those of $A / W$.

We begin with the simplest case, $s=1$.

Theorem 3.1. If $\boldsymbol{s}=1$ the torsion subgroup of $A / W$ is isomorphic to $\mathscr{T}$, the torsion subgroup of $A$, and the Betti number of $A / W$ is one less than that of A.

Proof. If $\mathscr{B}$ is a Betti subgroup of $A$, Lemma 3.1 (i) implies that there exists a base $\left(u_{1}, \cdots, u_{r}\right)$ of $\mathscr{B}$ such that $\boldsymbol{w}=u_{B}+\tau_{B}$, where $u_{B}=u_{1}$. A new Betti group $\mathscr{B}^{\prime}$, complementary to $\mathscr{T}$, can be introduced with a base

$$
\left(v_{1}, \cdots, v_{r}\right)=\left(u_{B}+\tau_{B}, u_{2}, \cdots, u_{r}\right)
$$


Then $\boldsymbol{w}=v_{1}$ and $A / W$ has the form

$$
\frac{A}{W}=\frac{\left\{v_{1}\right\} \oplus\left\{v_{2}\right\} \oplus \cdots \oplus\left\{v_{r}\right\} \oplus \mathscr{T}}{\left\{v_{1}\right\} \oplus\{0\} \oplus \cdots \oplus\{0\} \oplus\{0\}}
$$

of a "correlated" quotient. We infer from (2.3) that

$$
\frac{A}{W} \approx\left\{v_{2}\right\} \oplus \cdots \oplus\left\{v_{r}\right\} \oplus \mathscr{T} .
$$

Theorem 3.1 follows.

The case $s \geq 1$. The data. $A$ is "given" as is $A$ in $\S 2$. $A$ prescribed Betti group $\mathscr{B}$ of $A$ has a base $\left(u_{1}, \cdots, u_{r}\right)$ such that when $s \geq 1$

$$
\boldsymbol{w}=\boldsymbol{s} u_{B}+\tau_{B} \quad\left(u_{B}=u_{1}, \tau_{B} \in \mathscr{T}\right) .
$$

We begin with the case $\mathscr{T} \neq 0$, and suppose that $\mathscr{T}$ has a $C P D$,

$$
\mathscr{T}=\left\{x_{1}\right\} \oplus \cdots \oplus\left\{x_{\rho}\right\} \quad(\rho>0)
$$

for which the orders of the respective generators $x_{i}$ are the elementary divisors

$$
n_{1}, \cdots, n_{\rho}
$$

of $\mathscr{T}$. The element $\tau_{B}$ has a representation

$$
\tau_{B}=m_{1} x_{1}+\cdots+m_{\rho} x_{\rho}
$$

with

$$
0 \leq m_{i}<n_{i} \quad(i=1,2, \cdots, \rho) .
$$

We say that the set $\left(m_{1}, \cdots, m_{\rho}\right)$ is coordinated with the set $\left(n_{1}, \cdots, n_{\rho}\right)$ in that $m_{i}$ multiplies $x_{i}$ in (3.17), while $n_{i}$ is the order of $x_{i}$.

The set $\left(m_{1}, \cdots, m_{\rho}\right)$ depends upon the choice of $\mathscr{B}$, upon the ordering of the summands in (3.15) and upon the choice of the generators $x_{1}, \cdots, x_{\rho}$, as well as upon $\tau_{B}$. By rechoosing the generators $x_{i}$ we shall, in $\S 4$, obtain sets $\left(m_{1}, \cdots, m_{\rho}\right)$, termed canonical, such that $m_{i}=0$ or divides $n_{i}$.

Theorem 3.2, below, and Corollary 3.1 reduce the problem of determining the invariants of $A / W$ to the problem of determining the invariant factors of a $(\rho+1)$-square matrix with a diagonal,

$$
n_{1}, n_{2}, \cdots, n_{\rho}, \boldsymbol{s}
$$

and a last row, 


$$
m_{1}, m_{2}, \cdots, m_{\rho}, \boldsymbol{s},
$$

with other elements 0 .

Theorem 3.2 and Corollary 3.1 include the case $s=1$ even though the case $\boldsymbol{s}=1$ has already been disposed of in a simpler way,

Theorem 3.2. The case $s \geq 1, \mathscr{T} \neq 0$. If the data for $A$ and $\boldsymbol{w}$ are conditioned as above, $A / W$ has a Betti number, one less than that of $A$, and $a$ torsion subgroup $\hat{T}$ isomorphic to a quotient group $\boldsymbol{A} / \boldsymbol{R}$, where $\boldsymbol{A}$ is a free group,

$$
\boldsymbol{A}=\left\{y_{1}\right\} \oplus \cdots \oplus\left\{y_{\rho}\right\} \oplus\{v\}
$$

and $\boldsymbol{R}$ a subgroup of $\boldsymbol{A}$ of the form

$$
\boldsymbol{R}=\left\{n_{1} y_{1}, \cdots, n_{\rho} y_{\rho} ; \boldsymbol{s} v+m_{1} y_{1}+\cdots+m_{\rho} y_{\rho}\right\} .
$$

In proving Theorem 3.2 we shall make use of several lemmas. The "naturals) homomorphism"

$$
\Psi: A \longrightarrow A / W
$$

with its kernel $W=\{\boldsymbol{w}\}$ is needed. [10] p. 26 .

Lemma 3.2. When $s \geq 1$, the "natural homomorphism" $\Psi$ maps the torsion subgroup $\mathscr{T}$ of $A$ isomorphically onto the subgroup $\Psi(\mathscr{T})$ of $A / W$, and maps the subgroup

$$
\mathscr{B}_{1}=\left\{u_{2}\right\} \oplus \cdots \oplus\left\{u_{r}\right\}
$$

of $\mathscr{B}$ isomorphically onto $\Psi\left(\mathscr{B}_{1}\right)$.

Proof. Lemma 3.2 follows on noting that when $s \geq 1$

$$
\operatorname{ker}(\Psi \mid \mathscr{T})=\mathscr{T} \cap \operatorname{ker} \Psi=\mathscr{T} \cap\{\boldsymbol{w}\}=0 .
$$

since each non-trivial element of $\{\boldsymbol{w}\}$ then has an infinite order, and noting that

$$
\operatorname{ker}\left(\Psi \mid \mathscr{B}_{1}\right)=\mathscr{B}_{1} \cap \operatorname{ker} \Psi=\mathscr{B}_{1} \cap\{\boldsymbol{w}\}=0 .
$$

Lemma 3.3. When $s \geq 1$, the group $A / W$ is a direct sum,

$$
A / W=\Psi\left(\mathscr{B}_{1}\right) \oplus \Psi\left\{u_{1}, \mathscr{T}\right\}=\hat{B} \oplus \hat{T} \quad\left(u_{1} \text { from }(3.14)\right)
$$

6) The "natural" homomorphism $\Psi$ maps an element $x \in A$ into the element in $A / W$ which represents the $W$-coset containing $x$. 
in which $\Psi\left\{u_{1}, \mathscr{T}\right\}$ is the torsion subgroup $\hat{T}$ of $A / W$, and $\Psi\left(\mathscr{B}_{1}\right)$ is a Betti subgroup $\hat{B}$ of $A / W$.

To establish Lemma 3.3 it is sufficient to recall that $\Psi(A)=A / W$ and to verify successively the relations,

$$
\begin{gathered}
A=\left\{u_{1}, \mathscr{B}_{1}, \mathscr{T}\right\} \\
\Psi(A)=\left\{\Psi\left(u_{1}\right), \Psi\left(\mathscr{B}_{1}\right), \Psi(\mathscr{T})\right\} \\
\hat{T} \supset\left\{\Psi\left(u_{1}\right), \Psi(\mathscr{T})\right\} \\
0=\Psi\left(\mathscr{B}_{1}\right) \cap \hat{T}
\end{gathered}
$$

from which Lemma 3.3 follows.

Of these relations the first two are immediately verified.

Proof of (3.28). When $s \geq 1$

$$
\boldsymbol{w}=\boldsymbol{s} u_{B}+\tau_{B} \quad\left(u_{B}=u_{1}\right) .
$$

Recall that order $\tau_{B}$ in $\mathscr{T}$ has been denoted by $t_{B}$. The application of $\Psi$ to both members of (3.30) shows that

$$
0=\boldsymbol{s} \Psi\left(u_{1}\right)+\Psi\left(\tau_{B}\right) .
$$

Since order $\Psi\left(\tau_{B}\right)$ divides order $\tau_{B}$, we see that $s t_{B}$ annihilates $\Psi\left(u_{1}\right)$. Hence $\Psi\left(u_{1}\right)$ is in $\hat{T}$ and (3.28) follows.

Proof of (3.29). Relation (3.29) holds, since each element in $\hat{T}$ has a finite order and each element in $\Psi\left(\mathscr{B}_{1}\right)$, other than 0 , has an infinite order.

Lemma 3.3 follows.

We refer to the torsion subgroup $\hat{T}$ of $A / W$.

Lemma 3.4. When $s \geq 1$, there is a surjective isomorphism

$$
\hat{T} \approx \frac{\left\{u_{1}\right\} \oplus \mathscr{T}}{W}
$$

in which $W$-cosets in $A$ which are elements of $\hat{T}$ are mapped into the $W$-cosets in $\left\{u_{1}\right\} \oplus \mathscr{T}^{-}$partitioning $\left\{u_{1}\right\} \oplus \mathscr{T}$ with which they are identical as subsets of $A$.

Proof. Recall that $\hat{T}=\Psi\left\{u_{1}, \mathscr{T}\right\}$ and note that, as subsets of $A$,

$$
\left\{u_{1}, \mathscr{T}\right\}=\left\{u_{1}\right\} \oplus \mathscr{T} .
$$


Moreover $W$ is a subgroup, both of $A$ and of $\left\{u_{1}\right\} \oplus \mathscr{T}$ and $\hat{T}$ is the group of $W$-cosets in $A$ of elements in $\left\{u_{1}, \mathscr{T}\right\}$. Lemma 3.4 follows.

Notation for Lemma 3.5. Lemma 3.5, below, gives the final isomorphism leading to the isomorphism $\hat{T} \approx \boldsymbol{A} / \boldsymbol{R}$ of Theorem 3.2. In Lemma 3.5

$\mathscr{T}$ is given by a $C P D$ as in (3.15).

$\boldsymbol{A}$ is the free group (3.19)

$W$ is the subgroup $\left\{s u_{1}+m_{1} x_{1}+\cdots+m_{\rho} x_{\rho}\right\}$ of $A$

$\boldsymbol{R}$ is the subgroup (3.20) of $\boldsymbol{A}$

The statement and proof of Lemma 3.5 will be abbreviated by setting

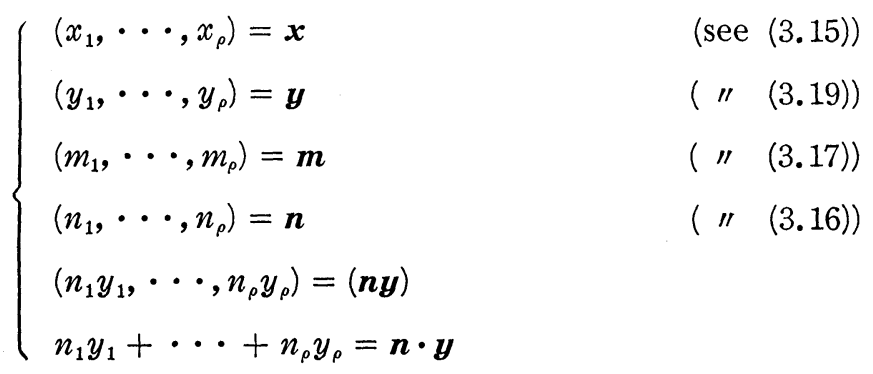

etc. We term $\boldsymbol{x}$ and $\boldsymbol{y}$ element sets. The set $(\boldsymbol{n} \boldsymbol{y})$ is an element set. We shall introduce arbitrary sets of integers,

$$
\begin{aligned}
& \left(q_{1}, \cdots, q_{\rho}\right)=\boldsymbol{q} \\
& \left(r_{1}, \cdots, r_{\rho}\right)=\boldsymbol{r}
\end{aligned}
$$

The cosets $Q(p, \boldsymbol{q})$ and $P(p, \boldsymbol{q})$. For arbitrary integer $p$ and set $\boldsymbol{q}$ of $\rho$ integers, we shall denote by $\boldsymbol{Q}(p, \boldsymbol{q})$, the $\boldsymbol{R}$-coset in $\boldsymbol{A}$ of the element $p v+\boldsymbol{q} \cdot \boldsymbol{y}$ in $\boldsymbol{A}$. We similarly denote by $P(p, \boldsymbol{q})$, the $W$-coset in $\left\{u_{1}\right\} \oplus \mathscr{T}$ of the element $p u_{1}+\boldsymbol{q} \cdot \boldsymbol{x}$ in $\left\{u_{1}\right\} \oplus \mathscr{T}$.

Lemma 3.5. When $s \geq 1$ there exists a surjective isomorphism

$$
\Theta: \frac{\boldsymbol{A}}{\boldsymbol{R}} \approx \frac{\left\{u_{1}\right\} \oplus \mathscr{T}}{W}
$$

in which the $\boldsymbol{R}$-coset, $Q(p, \boldsymbol{q})$ in $\boldsymbol{A}$, goes into the $W$-coset, $P(p, \boldsymbol{q})$ in $\left\{u_{1}\right\} \oplus \mathscr{T}$.

To prove Lemma 3.5 we note first that $\Theta$ is a homomorphism. That is, for arbitrary integers $p$ and $p^{\prime}$ and arbitrary sets $\boldsymbol{q}$ and $\boldsymbol{q}^{\prime}$ of $\rho$ integers

$$
\Theta\left(Q(p, \boldsymbol{q})+Q\left(p^{\prime}, \boldsymbol{q}^{\prime}\right)\right)=\Theta(Q(p, \boldsymbol{q}))+\Theta\left(Q\left(p^{\prime}, \boldsymbol{q}^{\prime}\right)\right) .
$$


The mapping $\Theta$ is surjective, since the ensemble of $W$-cosets $P(p, \boldsymbol{q})$ contains each $W$-coset in $\left\{u_{1}\right\} \oplus \mathscr{T}$. It ts crucial to establish Prop 3.1.

Proposition 3.1. The mapping $\Theta$ is biunique.

Note first that, if $\mathbf{0}$ is a set of $\rho$ zeros, then

$$
P(0, \mathbf{0})=W, \quad Q(0, \mathbf{0})=\boldsymbol{R} .
$$

Let $Q(p, \boldsymbol{q})$ and $Q\left(p^{\prime}, \boldsymbol{q}^{\prime}\right)$ be arbitrary distinct $\boldsymbol{R}$-cosets in $\boldsymbol{A}$. To establish Prop 3.1 we must show that the equality

$$
P(p, \boldsymbol{q})=P\left(p^{\prime}, \boldsymbol{q}^{\prime}\right)
$$

of the $\Theta$-images of $Q(p, \boldsymbol{q})$ and $Q\left(p^{\prime}, \boldsymbol{q}^{\prime}\right)$ is then impossible.

The equality (3.38) would imply that

$$
P\left(p-p^{\prime}, \boldsymbol{q}-\boldsymbol{q}^{\prime}\right)=P(0, \mathbf{0}),
$$

and hence that

$$
\Theta\left(Q\left(p-p^{\prime}, \boldsymbol{q}-\boldsymbol{q}^{\prime}\right)\right)=P(0, \mathbf{0}) .
$$

Moreover the hypothesis

$$
Q(p, \boldsymbol{q}) \neq Q\left(p^{\prime}, \boldsymbol{q}^{\prime}\right)
$$

implies that

$$
Q\left(p-p^{\prime}, \boldsymbol{q}-\boldsymbol{q}^{\prime}\right) \neq Q(0, \boldsymbol{0}) .
$$

Prop 3.2 below implies that (3.40) and (3.42) are incompatible, and hence that Prop 3.1 is true.

Let $\hat{p}$ be an arbitrary integer and $\hat{\boldsymbol{q}}$ an arbitrary set of $\rho$ integers.

Proposition 3.2. The only $\boldsymbol{R}$-coset $Q(\hat{p}, \hat{\boldsymbol{q}})$ in $\boldsymbol{A}$ whose image under $\Theta$ is the $W$-coset $P(0,0)$ in $\left\{u_{1}\right\} \oplus \mathscr{T}$ is the $\boldsymbol{R}$-coset $Q(0,0)$ in $\boldsymbol{A}$.

Proof of Prop 3.2. The $\Theta$-image of $Q(\hat{p}, \hat{\boldsymbol{q}})$ is $P(\hat{p}, \hat{\boldsymbol{q}})$ by definition of $\Theta$, and by hypothesis of Prop 3.2

$$
P(\hat{p}, \hat{\boldsymbol{q}})=P(0, \mathbf{0})=W .
$$

For (3.43) to hold it is necessary that for some integer $N$

$$
\hat{p} u_{1}+\hat{\boldsymbol{q}} \cdot \boldsymbol{x}=N\left(\boldsymbol{s} u_{1}+\boldsymbol{m} \cdot \boldsymbol{x}\right)
$$

since $\boldsymbol{s} u_{1}+\boldsymbol{m} \cdot \boldsymbol{x}$ is a generator of the cyclic group $W$. Since 


$$
\left\{u_{1}\right\} \oplus \cdots \oplus\left\{u_{r}\right\} \oplus\left\{x_{1}\right\} \oplus \cdots \oplus\left\{x_{\rho}\right\}
$$

is a direct sum, and order $x_{i}=n_{i}$ for $i=1, \cdots, \rho$, (3.44) holds only if

$$
\hat{p}=N s, \hat{\boldsymbol{q}}=N \boldsymbol{m}+(\boldsymbol{r n})
$$

for some set $\boldsymbol{r}$ of $\rho$ integers. It follows from (3.46) that

$$
\hat{p} v+\hat{\boldsymbol{q}} \cdot \boldsymbol{y}=N(\boldsymbol{s} v+\boldsymbol{m} \cdot \boldsymbol{y})+(\boldsymbol{r n}) \cdot \boldsymbol{y},
$$

that is, that $\hat{p} v+\hat{\boldsymbol{q}} \cdot \boldsymbol{y}$ is in $\boldsymbol{R}$.

Thus Prop 3.2 is true, $\Theta$ is biunique, and Lemma 3.5 follows.

Completion of proof of Theorem 3.2. It follows from Lemmas 3.2 and 3.3 that, when $s \geq 1$, the Betti number of $A / W$ is one less than the Betti number of $A$. That the torsion subgroup $\hat{T}$ of $A / W$ is isomorphic to the quotient $\boldsymbol{A} / \boldsymbol{R}$ of Theorem 3.2 follows from Lemmas 3.4 and 3.5.

Theorem 3.2 is thereby established. Theorem 3.2 is supplemented as follows.

The case $\mathscr{T}=0$. In stating and proving Theorem 3.2 we have excluded this special case. In this case $\tau_{B}=0$ in (3.14), so that

$$
\frac{A}{W}=\frac{\left\{u_{1}\right\} \oplus\left\{u_{2}\right\} \oplus \cdots \oplus\left\{u_{r}\right\}}{\left\{s u_{1}\right\} \oplus\{0\} \oplus \cdots \oplus\{0\}} .
$$

This is a "correlated" quotient. By (2.3)

$$
\frac{A}{W} \approx C(s) \oplus\left\{u_{2}\right\} \oplus \cdots \oplus\left\{u_{r}\right\} \quad \text { (when } s>1 \text { ) }
$$

where $C(\boldsymbol{s})$ is a cyclic group of order $\boldsymbol{s}$. The torsion subgroup of $A / W$ is thus isomorphic to $C(\boldsymbol{s})$ when $\mathscr{T}=0$ and $\boldsymbol{s}>1$, and trivial when $\boldsymbol{s}$ is 1 or 0 .

From (3.47) and (3.48) one can infer the following:

Theorem 3.3. When $A$ is torsion free $A / W$ is torsion free unless $\boldsymbol{s}>1$, and when $\boldsymbol{s}>1$ the first and only torsion coefficient of $A / W$ is $\boldsymbol{s}$.

The torsion coefficients of $A / W$ when $s \geq 1$. The subgroup $\boldsymbol{R}$ of the free group $\boldsymbol{A}$ is defined by a set of generators $z_{1}, \cdots, z_{\rho+1}$ given in Theorem 3.2 as the respective linear combinations,

$$
n_{1} y_{1}, \cdots, n_{\rho} y_{\rho}, \quad s v+m_{1} y_{1}+\cdots+m_{\rho} y_{\rho}
$$

of the elements 


$$
y_{1}, \cdots, y_{\rho}, v
$$

of the base of $\boldsymbol{A}$. Cf. (2.3), (2.4) and (2.5). The matrix $\left\|a_{i j}\right\|$ of the integral linear representation of $z_{1}, \cdots, z_{\rho+1}$ in terms of the elements (3.50) has the form

$$
\left\|a_{i j}\right\|=\left\|\begin{array}{c}
n_{1} n_{2} \cdot n_{\rho} \\
m_{1} m_{2} \cdots m_{\rho} s
\end{array}\right\| \quad(\boldsymbol{s} \geq 1)
$$

where unspecified elements are 0 .

The rank of the matrix (3.51) is $\rho+1$. The integers $m_{1}, \cdots, m_{\rho}$ were introduced in (3.17). They satisfy the conditions

$$
0 \leq m_{i}<n_{i} \quad(i=1, \cdots, \rho)
$$

and are "coordinated" with the elementary divisors $n_{1}, \cdots, n_{\rho}$ of $A$.

From Theorem 2.1 we infer a fundamental corollary of Theorem 3.2.

Corollary 3.1. When $s \geq 1$ the rank of the matrix $\left\|a_{i j}\right\|$ is $\rho+1$ and the subset of invariant factors of this matrix which exceed 1 are the torsion coefficients of $A / W$.

Making use of this corollary it is possible to make many comparisons between the elementary divisors of $A$ and those of $A / W$ when $s \geq 1$, or equivalently the torsion coefficients of $A$ and those of $A / W$.

A first result of this type follows.

Corollary 3.2. Suppose $s \geq 1$.

(i) If each $m_{i}$ in the representation (3.17) of $\varepsilon_{B}$ is a multiple (possibly null) of $\boldsymbol{s}$, then the torsion coefficients of $A / W$ are the invariant factors which exceed 1 of the diagonal matrix $J$ with diagonal

$$
n_{1}, \cdots, n_{\rho}, s \text {. }
$$

(ii) Equivalently, under the hypotheses of (i) the elementary divisors of $A / W$ are those of $A$ supplemented by the prime power factors of $\boldsymbol{s}$.

Proof of (i). Matrices which can be obtained, one from the other, by elementary operations are termed equivalent. Equivalent matrices have the same invariant factors. 
It is clear that under the hypotheses of (i), the matrices $\left\|a_{i j}\right\|$ and $J$ are equivalent.

Statement (i) follows.

Proof of (ii). There exists an abstract abelian group $A^{*}$ which is the external direct sum of abelian groups with the orders (3.53). By Corollary 2.1 , the torsion coefficients of $A^{*}$ are the invariant factors exceeding one of the matrix $J$, and hence by (i) the torsion coefficients of $A / W$. The elementary divisors of $A^{*}$ are clearly the integers $n_{1}, \cdots, n_{\rho}$, supplemented by the prime power factors of $\boldsymbol{s}$. Since $A^{*}$ and $A / W$ have the same torsion coefficients they have the same elementary divisors.

Statement (ii) follows.

In case $s \geq 1$ the quotient $A / W$ has more torsion than $A$ in the sense of the following corollary.

Corollary 3.3. When $s \geq 1$ the product of the torsion coefficients of $A / W$ (or elementary divisors of $A / W$ ) is $\boldsymbol{s}$ times the corresponding product for $A$.

Proof. We have seen in $\S 1$ that the product of the elementary divisors of $A$ equals the product of the torsion coefficients of $A$. Cf. Theorem 1.3. In the case at hand this product is $n_{1} \cdots n_{\rho}$ by hypothesis. According to Corollary 3.1 the product, when $s \geq 1$, of the torsion coefficients of $A / W$ is the product of the invariant factors of the matrix $\left\|a_{i j}\right\|$. Hence this product equals

$$
\operatorname{det}\left|a_{i j}\right|=n_{1} \cdots n_{\rho} s .
$$

Thus Corollary 3.3 is true.

In $\S 5$ an explicit comparison of the elementary divisors of $A$ and $A / W$ is made when both $A$ and $A / W$ are prime-simple groups. See Definition 1.6.

$\S 4$. The elementary quotients $A / W$ when $s=0$. We continue with the analysis of $\S 3$. As in $\S 3, A$ is finitely generated, $W$ a subgroup of $A$ of form $W=\{\boldsymbol{w}\}$, with $\boldsymbol{w} \neq 0$ in $\mathscr{T}$, the torsion subgroup of $A$.

We begin with a lemma.

Lemma 4.1. If $\boldsymbol{s}=0$ the Betti number of $A / W$ equals the Betti number of $A$, and if $T_{0}$ is the torsion subgroup of $A / W$, then

$$
T_{0} \approx \mathscr{T} / W
$$


Let $\mathscr{B}$ be a Betti subgroup of $A$. Then $A=\mathscr{B} \oplus \mathscr{T}$ and one has the correlated quotient

$$
\frac{A}{W}=\frac{\mathscr{B} \oplus \mathscr{T}}{\{0\} \oplus W}, \quad(s=0)
$$

since $W$ is a subgroup of $\mathscr{T}$ when $\boldsymbol{s}=0 . \quad$ By (2.3)

$$
A / W \approx \mathscr{B} \oplus \mathscr{T} / W .
$$

The quotient $\mathscr{T} / W$ is a finite group and hence the torsion subgroup of the right member of (4.2). The isomorphism (4.0) follows from the isomorphism (4.2).

Note. One should contrast the equality of the Betti numbers of $A$ and $A / W$ when $s=0$, with the fact that the Betti number of $A$ exceeds the Betti number of $A / W$ by 1 when $s>0$. See Theorems 3.1 and 3.2.

We now prepare for Theorem 4.1.

The data when $s=0 . \quad A$ is given as is $A$ of (2.1). Since $s=0$

$$
\boldsymbol{w}=\tau_{B} \quad\left(\tau_{B} \in \mathscr{T}\right),
$$

where $\tau_{B} \neq 0$, since we are assuming that $W=\{\boldsymbol{w}\} \neq 0$. We suppose that $\mathscr{T}$ is given by a $C P D$ of form (3.15) and that $\left(n_{1}, \cdots, n_{\rho}\right)$ is the corresponding set of ${ }^{7)} E D$ 's of $\mathscr{T}$. We suppose that $\tau_{B}$ is given a representation (3.17) with a set of integral coefficients $\left(m_{1}, \cdots, m_{\rho}\right)$ subject to (3.18), and "coordinated" with the ordered set $\left(n_{1}, \cdots, n_{\rho}\right)$ of $E D$ 's of $\mathscr{T}$.

Theorem 4.1. The case $s=0$. Under the conditions of the preceding paragraph the torsion subgroup $T_{0}$ of $A / W$ is isomorphic to a quotient group $A_{0} / R_{0}$, where $A_{0}$ is a free group,

$$
A_{0}=\left\{y_{1}\right\} \oplus \cdots \oplus\left\{y_{\rho}\right\},
$$

and $R_{0}$ is a subgroup of $A_{0}$ of the form

$$
R_{0}=\left\{n_{1} y_{1}, \cdots, n_{\rho} y_{\rho} ; m_{1} y_{1}+\cdots+m_{\rho} u_{\rho}\right\} .
$$

Proof. By virtue of Lemma 4.1, it is sufficient to show that

$$
\frac{\mathscr{T}}{W} \approx \frac{A_{0}}{R_{0}}
$$

Proof of (4.5). For arbitrary sets $\boldsymbol{q}$ of $\rho$ integers, let $Q(\boldsymbol{q})$ denote the

7) $E D$ abbreviates "elementary divisor". 
$R_{0}$-coset in $A_{0}$ of the element $\boldsymbol{q} \cdot \boldsymbol{y}$ in $A_{0}$ (employing the notation introduced in (3.34)). Similarly let $P(\boldsymbol{q})$ denote the $W$-coset in $\mathscr{T}$ of the element $\boldsymbol{q} \cdot \boldsymbol{x}$ in $\mathscr{T}$. Let $\Phi$ be the surjective homomorphism,

$$
\Phi: Q(\boldsymbol{q}) \longrightarrow P(\boldsymbol{q}): \frac{A_{0}}{R_{0}} \longrightarrow \frac{\mathscr{T}}{W} .
$$

As in the proof of Theorem 3.2 the principal step is to prove $\Phi$ biunique. Just as Proposition 3.1 followed from Proposition 3.2, so here the biuniqueness of $\Phi$ follows from the analogue, Proposition 4.1, of Proposition 3.2.

Proposition 4.1. The only $R_{0}$-coset, $Q(\hat{\boldsymbol{q}})$ in $A_{0}$, whose image under $\Phi$ is the $W$-coset $P(\mathbf{0})$ in $\mathscr{T}$, is the $R_{0}$-coset $Q(\mathbf{0})$ in $A_{0}$.

Proof of Proposition 4.1. As in the proof of Proposition 3.2, by definition and hypothesis,

$$
\Phi(Q(\hat{\boldsymbol{q}}))=P(\hat{\boldsymbol{q}})=P(\mathbf{0})=\{\boldsymbol{w}\} .
$$

For (4.7) to hold, it is necessary that for some integer $N$

$$
\hat{\boldsymbol{q}} \cdot \boldsymbol{x}=N(\boldsymbol{m} \cdot \boldsymbol{x})
$$

since $\boldsymbol{m} \cdot \boldsymbol{x}=\boldsymbol{w}$ by (3.17) and (3.14). The relation (4.8) implies that

$$
\hat{\boldsymbol{q}}=N \boldsymbol{m}+(\boldsymbol{r n})
$$

for some set $\boldsymbol{r}$ of $\rho$ integers. It follows from (4.9) that

$$
\hat{\boldsymbol{q}} \cdot \boldsymbol{y}=N(\boldsymbol{m} \cdot \boldsymbol{y})+(\boldsymbol{r} \boldsymbol{n}) \cdot \boldsymbol{y},
$$

that is, that $\hat{\boldsymbol{q}} \cdot \boldsymbol{y}$ is in $R_{0}$.

Thus Proposition 4.1 is true, hence $\Phi$ is biunique. It is a surjective isomorphism.

This completes the proof of Theorem 4.1.

The torsion coefficients of $A / W$ when $s=0$. The subgroup $R_{0}$ of the free group $A_{0}$ of Theorem 4.1 is defined by a set of generators $z_{1}, \cdots, z_{\rho+1}$, given in Theorem 4.1 as the respective linear combinations

$$
n_{1} y_{1}, \cdots, n_{\rho} y_{\rho} ; m_{1} \dot{y}_{1}+\cdots+m_{\rho} y_{\rho}
$$

of the elements $y_{1}, \cdots, y_{\rho}$ of the base of $A_{0}$. The matrix $\left\|b_{i j}\right\|$ of these integral linear combinations of the elements $y_{1}, \cdots, y_{\rho}$, has the form 


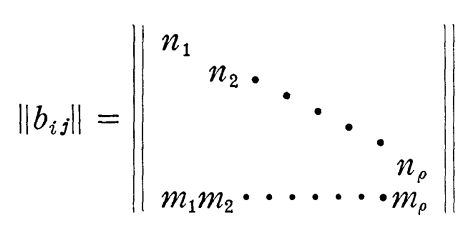

where unspecified elements are 0 .

From Theorem 2.1 we infer the following corollary of Theorem 4.1.

Corollary 4.1. When $s=0$ the rank of the matrix $\left\|b_{i j}\right\|$ is $\rho$ and the invariant factors of $\left\|b_{i j}\right\|$ which exceed 1 are the torsion coefficients of $A_{0} / R_{0}$ and hence of $A / W$.

Canonical sets $\left(m_{1}, \cdots, m_{\rho}\right)$. We refer to the representation

$$
\tau_{B}=m_{1} x_{1}+\cdots+m_{\rho} x_{\rho}
$$

$$
\left(\tau_{B} \in \mathscr{T}\right)
$$

of $\tau_{B}$. The coefficients $m_{i}$ which are not zero will be altered, in general, by a change of the generators $x_{i}$ of the summands $\left\{x_{i}\right\}$ of the $C P D$ (3.15) of $\mathscr{T}$. However, one does not alter $\left\{x_{i}\right\}$ if $x_{i}$ is replaced by $r x_{i}$, provided $r$ is relatively prime to order $\left\{x_{i}\right\}$. The following lemma shows how this freedom of choice of the generators can simplify the set $\left(m_{1}, \cdots, m_{\rho}\right)$.

Lemma 4.2. If generators $x_{i}$ of the summands $\left\{x_{i}\right\}$ of the CPD (3.15) of $\tau_{B}$ are suitably chosen, each coefficient, $m_{i} \neq 0$, in the representation (4.12) of $\tau_{B}$ will become a proper divisor (termed canonical) of $n_{i}$, the associated ED of $\mathscr{T}$ and as such will be unique.

Proof. The $E D n_{i}$ equals $p_{i}^{e_{i}}$ for some prime factor $p_{i}$ of order $\mathscr{T}$ and positive integer $e_{i}$. If $m_{i} \neq 0$ there exists a $G C D$

$$
\left(n_{i}, m_{i}\right)=p_{i}^{a_{i}}
$$

of $n_{i}$ and $m_{i}$, such that $0 \leq a_{i}<e_{i}$. Hence $m_{i}=\mu_{i} p_{i}^{a_{i}}$, where $\mu_{i}$ and $p_{i}$ are relatively prime. The generator $x_{i}$ can be replaced by a generator $x_{i}^{\prime}=\mu_{i} x_{i}$. Then in (4.12) one has

$$
m_{i} x_{i}=\mu_{i} p_{i}^{a_{i}} x_{i}=x_{\imath}^{\prime} p_{i}^{a_{i}} \quad \text { (with } p_{i}^{a_{2}} \text { canonical). }
$$

so that one can replace $x_{i}$ by $x_{i}^{\prime}$ as generator of $\left\{x_{i}\right\}$ and $m_{i}$ by $m_{i}^{\prime}=p_{i}^{a_{i}}$ in (3.17).

When $m_{i} \neq 0$, and $p_{i}^{a_{i}}$ is conditioned as above, $m_{i}=\mu_{i} p_{i}^{a_{i}}$ where $\mu_{i}$ is prime to $p_{i}$ so that 


$$
\text { order }\left(m_{i} x_{i}\right)=\text { order }\left(\mu_{i} p_{i}^{a_{i}} x_{i}\right)=\operatorname{order} p_{i}^{a_{i}} x_{i}=p_{i}^{e_{i}-a_{i}} .
$$

Hence the replacement $m_{i}^{\prime}=p_{i}^{a_{i}}$ of $m_{i}$ is uniquely determined by the requirement that $\left\{x_{i}\right\}=\left\{x_{i}^{\prime}\right\}, m_{i} x_{i}=m_{i}^{\prime} x_{i}^{\prime}$ and that $m_{i}^{\prime}$ be a proper divisor of $n_{i}$.

The computation of the $E D$ 's of $A / W$ when $s=0 . \quad$ As has been seen in $\S 1$ the $E D$ 's of $A / W$ are immediate if the torsion coefficients of $A / W$ are known. According to Corollary 4.1, when $s=0$ the torsion coefficients of $A / W$ are the invariant factors exceeding 1 of the matrix $\left\|b_{i j}\right\|$ of (4.11). However, this computation of the $E D$ 's of $A / W$ can be simplified as follows.

Let $p_{1}, \cdots, p_{r}$ be the distinct primes which are factors of (order $\mathscr{T}$ ) when $s=0$. The subgroup $W$ of $\mathscr{T}$ can be given a Sylow decomposition $W=W_{1} \oplus \cdots \oplus W_{r}$ into a direct sum of the Sylow $p_{i}$-subgroups $W_{i}$ of $W$, some of which can be trivial. If $\mathscr{S}_{p_{i}}$ is the Sylow $p_{i}$-subgroup of $\mathscr{T}$ then

$$
\frac{\mathscr{T}}{W} \approx \frac{\mathscr{S}_{p_{1}}}{W_{1}} \oplus \cdots \oplus \frac{\mathscr{S}_{p_{r}}}{W_{r}}
$$

We infer the following.

Lemma 4.3. When $\boldsymbol{s}=0$ the set of $E D$ 's of $\mathscr{T} / W$ is the union of the $E D$ 's of such of the quotients $\mathscr{S}_{p_{\mu}} / W_{\mu}$ in (4.16) as are nontrivial groups.

Matrices $J_{\mu}$. We are accordingly led to the problem of computation of the torsion quotients of a non-trivial quotient $\mathscr{S}_{p_{\mu}} / W_{\mu}$ taken from the quotients in the right member of (4.16). With such a quotient we associate that submatrix $J_{\mu}$ of the matrix $\left\|b_{i j}\right\|$ which consists of the columns of $\left\|b_{i j}\right\|$ whose non-null elements are powers of $P_{\mu}$. If the rows of $J_{\mu}$ are properly rearranged $J_{\mu}$ will have the general form of $\left\|b_{i j}\right\|$ with a diagonal composed of $E D$ 's of $\mathscr{S}_{p_{\mu}}$, say $\omega$ in number, and an $(\omega+1)$-th row of integers $m_{1}, \cdots, m_{\omega}$ each of which is 0 or divides the $E D$ of $\mathscr{S}_{p_{\mu}}$ in the same column.

The invariant factors of $J_{\mu}$. The computation of the invariant factors $d_{1}, \cdots, d_{\omega}$ of $J_{\mu}$ is very simple by virtue of the fact that

$$
d_{1}=D_{1}, d_{2}=\frac{D_{2}}{D_{1}}, \cdots, d_{\omega}=\frac{D_{\omega}}{D_{\omega-1}}
$$

where for $i$ on the range, $1, \cdots, \omega, D_{i}$ is the smallest in absolute value of the non-vanishing $i$-rowed subdeterminants $\Delta_{i}$ of $J_{\mu}$. One observes that each non-vanishing $\Delta_{i}$ is the product of $i$ elements of $J_{\mu}$. 
Definition 4.1. Profiles of $\boldsymbol{w}$. If the generator $\boldsymbol{w}$ of $W$ is given as in (1.9), subject to (1.10), the set of integers

$$
\mu_{1}, \cdots, \mu_{\beta}, m_{1}, \cdots, m_{\rho}
$$

will be called the profile of $\boldsymbol{w}$ relative to the "basis" (1.8) of $A$.

We shall prove the following theorem.

Theorem 4.2. A "profile" of a non-null element $\boldsymbol{w} \in A$ uniquely determines the "free index" $s$, and together with the coordinated ED's of $\mathscr{T}$ uniquely determines the "torsion index" of $\boldsymbol{w}$ when $\boldsymbol{s}=0$.

To establish this theorem a second definition is needed.

Definition 4.2. If the generators $x_{i}$ of the summands of the $C P D$ of $\mathscr{T}$ are suitably chosen the non-null integers $m_{i}$ in the set $m_{1}, \cdots, m_{\rho}$ are "canonical" in the sense of Lemma 4.2, and the "profile" (4.18) relative to the basis (1.8) will be termed canonical.

According to Lemma 4.2 a profile (4.18) of $\boldsymbol{w}$ relative to a "basis" (1.8) uniquely determines a "canonical profile" of $\boldsymbol{w}$ relative to a modified basis (1.8). Theorem 4.2 is accordingly equivalent to the following lemma.

Lemma 4.4. A canonical profile

$$
\mu_{1}, \cdots, \mu_{\beta} ; m_{1}, \cdots, m_{\rho}
$$

of a non-null element $\boldsymbol{w} \in A$ uniquely determines the free index $\boldsymbol{s}$ of $\boldsymbol{w}$ and together with the coordinated ED's of $\mathscr{T}$ uniquely determines order $\boldsymbol{w}$ when $\boldsymbol{s}=0$.

The free index $s$. If $\beta=0$ in (4.19), or if $\mu_{1}=\cdots=\mu_{\beta}=0$, then $s=0$. In any other case $s$ is the $G C D$ of the elements $\mu_{1}, \cdots, \mu_{\beta}$, as the proof of Lemma 3.1(i) shows.

The torsion index $\boldsymbol{t}$. If $\boldsymbol{s}=0, \boldsymbol{t}=$ order $\boldsymbol{w}$ in accord with the definition of $\boldsymbol{t}$. If $\mathscr{T}=0$, or if $m_{1}=\cdots=m_{\rho}=0, \boldsymbol{t}=1$.

Suppose then that $\boldsymbol{s}=0$ and $\boldsymbol{w} \neq 0$. Let $n_{1}, \cdots, n_{\rho}$ be the ED's of $\mathscr{T}$.

Suppose first that $\mathscr{T}$ is a Sylow $p$-subgroup of $A$. The $E D$ 's of $\mathscr{T}$ then form a list

$$
p^{e_{1}}, \cdots, p^{e_{\rho}} \quad\left(e_{i}>0\right)
$$

of powers of $p$. By hypothesis (4.19) is a "canonical profile" of $\boldsymbol{w}$ relative 
to a basis (1.8) of $A$. We suppose that the $E D$ 's (4.20) are so ordered that for some integer $r$ such that $1 \leq r \leq \rho, m_{1}, \cdots, m_{r}$ is the subset of the $m_{i}$ 's in (4.19) which do not vanish. Since (4.19) is by hypothesis a "canonical profile" of $\boldsymbol{w}$ the first $r$ elements in the reordered list $m_{1}, \cdots, m_{\rho}$ have the form

$$
p^{a_{1}}, \cdots, p^{a_{r}} \quad\left(0 \leq a_{j}<e_{j}\right)
$$

where $j$ has the range $1,2, \cdots, r$.

Moreover

$$
\boldsymbol{w}=p^{a}{ }_{1} x_{1}+\cdots+p^{a_{r}} x_{r} .
$$

We shall show that

$$
\text { order } \boldsymbol{w}=\max _{j} p^{e_{j}-a_{j}}=p^{e},
$$

introducing $p^{e}$.

Proof of (4.23). One establishes $(4.23)$ by verifying the following.

(a) The order of $p^{a_{j}} x_{j}$ in $\left\{x_{j}\right\}$ is $p^{e_{j}-a_{j}}$.

(b) The integer $p^{e}$ annihilates $\boldsymbol{w}$.

(c) The order $\boldsymbol{t}$ of $\boldsymbol{w}$ is a divisor $p^{a}$ of $p^{e}$.

(d) Were $0 \leq a<e, p^{a}$ could not annihilate $\boldsymbol{w}$.

Statements (a), (b) and (c) are readily verified. Were $a<e$ and $p^{a}$ an annihilator of $\boldsymbol{w}$ it would follow from the representation (4.22) of $(\boldsymbol{w})$ that

$$
\left\{x_{1}, \cdots, x_{r}\right\}
$$

could not be a direct sum $\left\{x_{1}\right\} \oplus \cdots \oplus\left\{x_{r}\right\}$. From this contradiction (d) follows.

Thus (4.23) is true and Lemma 4.4 follows when $\mathscr{T}$ is a Sylow $p$-subgroup of $A$.

In any case $\mathscr{T}$ is a direct sum of its Sylow subgroups. The order of $\boldsymbol{w}$ is then the product of the orders of its non-null components in the respective Sylow subgroups of $\mathscr{T}$.

Lemma 4.4 follows and implies Theorem 4.2.

§5. $A / W$ when $A$ is prime-simple. We shall give an explicit determination in this section of how the $E D$ 's of $A / W$ differ from those of 
$A$ when both $A$ and $A / W$ are prime-simple in the sense of Def 1.6. To this end a general lemma with no assumption of prime-simplicity will be useful.

Notation for Lemma 5.1. We refer again to the natural homomorphism $\Psi: A \longrightarrow A / W$ of $A$ onto $A / W$, where $W=\{\boldsymbol{w}\}$, and as in (3.8),

$$
\boldsymbol{w}=\boldsymbol{s} u_{B}+\tau_{B} \quad\left(\tau_{B} \in \mathscr{T}\right) .
$$

Here $u_{B}$ is an element in $A$ which is 0 or a first element in a base for a Betti group of $A$, according as $\boldsymbol{w}$ is in $\mathscr{T}$ or not in $\mathscr{T}$.

Lemma 5.1. If order $\tau_{B}$ in $A$ is denoted by $t_{B}$, order $\Psi\left(u_{B}\right)$ in $A / W$ equals st $t_{B}$ or 1 according as $u_{B} \neq 0$ or $u_{B}=0$.

The lemma is trivial if $u_{B}=0$.

Suppose then that $u_{B} \neq 0$ and $s>0$. Since $\operatorname{ker} \Psi=\{\boldsymbol{w}\}$ it follows from (5.1) that

$$
\Psi(\boldsymbol{w})=\boldsymbol{s} \Psi\left(u_{B}\right)+\Psi\left(\tau_{B}\right)=0 .
$$

Moreover, $t_{B} \Psi\left(\tau_{B}\right)=0$, since $t_{B} \tau_{B}=0$. Hence $s t_{B} \Psi\left(u_{B}\right)=0$, so that order $\Psi\left(u_{B}\right)$ is a divisor $m$ of $s t_{B}$.

Since $m \Psi\left(u_{B}\right)=0, m u_{B}$ must be in $\operatorname{ker} \Psi$. Hence

$$
m u_{B}=\mu\left(s u_{B}+\tau_{B}\right)
$$

for some integer $\mu$. Since $u_{B} \neq 0$ by hypothesis, $m=\mu \boldsymbol{s}$. Hence $\mu \tau_{B}=0$. It follows that $\mu$ is a multiple of $t_{B}$. We conclude that $m$ must be a multiple of $s t_{B}$ and so equal $s t_{B}$.

This establishes Lemma 5.1.

We now suppose that $A$ is prime-simple. When $A$ is finitely generated and $W=\{\boldsymbol{w}\}$, we have seen in Lemma 4.1 that the torsion subgroup, $T_{0}$ of $A / W$, when $s=0$, is such that

$$
T_{0} \approx \mathscr{T} / W
$$

while Lemma 3.4 implies that the torsion subgroup, $\hat{T}$ of $A / W$, when $s \geq 1$, is such that

$$
\hat{T} \approx \frac{\left\{u_{1}\right\} \oplus \mathscr{T}}{W}
$$

We include the case $s=1$, although this case is adequately covered by Theorem 3.1. 
Corresponding to the cases $\boldsymbol{s}=0$ and $\boldsymbol{s} \geq 1$, Theorems 5.1 and 5.2 are the principal results of this section. We are assuming that $A$ is finitely generated and that $W=\{\boldsymbol{w}\}$ is non-trivial. By hypothesis each $E D$ of $A$ is a prime.

$A$ convention. To say that a prime $p$ is an $E D$ of $A / W$ of multiplicity 0 shall mean that $p$ is not an $E D$ of $A / W$.

Theorem 5.1. When $\boldsymbol{s}=0$ and $A$ is prime-simple, each ED of $A$ which is not a factor of (order $\boldsymbol{w}$ ) is an $E D$ of $A / W$, while each $E D$ of $A$, of multiplicity $\mu$ which is a factor of (order $\boldsymbol{w}$ ) is an $E D$ of $A / W$ of multiplicity $\mu-1$.

These are the only $E D$ 's of $A / W$ and $A / W$ is accordingly prime-simple (Th 1.4).

We refer to the "torsion index" $t$ of $\boldsymbol{w}$. Def 3.2.

Theorem 5.2. When $s \geq 1$ and $A$ is prime-simple, the following is true:

(i) Necessary and sufficient conditions that $A / W$ be prime-simple are that $\boldsymbol{s}$ be prime-simple and that $\boldsymbol{t}=1$.

(ii) When $\boldsymbol{s}$ is prime-simple and $\boldsymbol{t}=1$, the ED's of $A$, supplemented by the prime factors of $\boldsymbol{s}$, are the $E D$ 's of $A / W$.

Proof of Theorem 5.1. It follows from Def 1.6 of a prime-simple group that each subgroup of a prime-simple group is prime-simple. Hence $W=\{\boldsymbol{w}\}$ is prime-simple. Since $\boldsymbol{s}=0$ by hypothesis, $\boldsymbol{w}$ has a finite order. Since $W$ is nontrivial by hypothesis and is finite and prime-simple, order $\boldsymbol{w}$ is a product

$$
p_{1} p_{2} \cdots p_{r} \quad(r>0)
$$

of distinct primes. Lemma 5.2 is needed.

Notation for Lemma 5.2. Let $p$ be one of the primes in the product (5.6). The Sylow subgroup $\mathscr{S}_{p}$ of $A$ is prime-simple since $A$ is primesimple. Let $\mu$ be the "length" of $\mathscr{S}_{p}$. Each $E D$ of $\mathscr{S}_{p}$ must equal $p$ in magnitude. $\mathscr{S}_{p}$ must then have a $C P D$ of the form

$$
\mathscr{S}_{p}=\left\{e_{1}\right\} \oplus \cdots \oplus\left\{e_{\mu}\right\}
$$

where each generator $e_{i}$ has the order $p$.

We shall verify the following lemma. 
Lemma 5.2. If an element $a \in W$ has the prime order $p$ and $\mathscr{S}_{p}$ has the length $\mu$, then the quotient group $\mathscr{S}_{p} /\{a\}$ has exactly $\mu-1 E D$ 's, each numerically $p$.

Proof. If the generators $e_{i}$ of the cyclic groups $\left\{e_{i}\right\}$ in (5.7) are properly chosen and ordered, then

$$
\left.a=e_{1}+e_{2}+\cdots+e_{n} \quad \text { (cf. Lemma } 4.2\right)
$$

for some integer $n$ such that $1 \leq n \leq \mu$. The subgroups

$$
\{a\},\left\{e_{2}\right\}, \cdots,\left\{e_{\mu}\right\}
$$

of $\mathscr{S}_{p}$ generate $\mathscr{S}_{p}$. They also sum directly to $\mathscr{S}_{p}$; for a nontrivial relation of integral linear dependence between the generators $a, e_{2}, \cdots, e_{\mu}$ would imply a nontrivial relation of integral linear dependence between the elements $e_{1}, \cdots, e_{\mu}$. Hence

$$
\frac{\mathscr{S}_{p}}{\{a\}}=\frac{\{a\} \oplus\left\{e_{2}\right\} \oplus \cdots \oplus\left\{e_{\mu}\right\}}{\{a\} \oplus\{0\} \oplus \cdots \oplus\{0\}}
$$

This is a "correlated quotient". It follows from (2.3) that

$$
\mathscr{S}_{p} /\{a\} \approx\left\{e_{2}\right\} \oplus \cdots \oplus\left\{e_{\mu}\right\},
$$

thereby establishing Lemma 5.2.

Completion of proof of Theorem 5.1. Starting with the primes (5.6) let

$$
p_{1}, \cdots, p_{r} ; p_{r+1}, \cdots, p_{m}
$$

be the list of distinct primes which are the $E D$ 's of the torsion subgroup $\mathscr{T}$ of $A$. The complete set of $E D$ 's of $\mathscr{T}$ is obtained by listing each of the primes in (5.11) a number of times equal to its multiplicity. One has the Sylow decomposition

$$
\mathscr{T}=\mathscr{S}_{p_{1}} \oplus \cdots \oplus \mathscr{S}_{p_{m}}
$$

of $\mathscr{T}$. The subgroup $W$ of $A$ admits a $C P D$

$$
W=\left\{a_{1}\right\} \oplus \cdots \oplus\left\{a_{r}\right\}
$$

where the generator $a_{i}$ has the order $p_{i}$. From (5.12) and (5.13) we obtain a correlated quotient 


$$
\frac{\mathscr{T}}{W}=\frac{\mathscr{S}_{p_{1}} \oplus \cdots \oplus \mathscr{S}_{p_{r}} \oplus \mathscr{S}_{p_{r+1}} \oplus \cdots \oplus \mathscr{S}_{p_{m}}}{\left\{a_{1}\right\} \oplus \cdots \oplus\left\{a_{r}\right\} \oplus\{0\} \oplus \cdots \oplus\{0\}}
$$

It follows from (5.14) and (5.4) that $T_{0}$ is isomorphic to the external direct sum

$$
\frac{\mathscr{S}_{p_{1}}}{\left\{a_{1}\right\}} \oplus \cdots \oplus \frac{\mathscr{S}_{p_{r}}}{\left\{a_{r}\right\}} \oplus \mathscr{S}_{p_{r+1}} \oplus \cdots \oplus \mathscr{S}_{p_{m}} .
$$

Theorem 5.1 follows on applying Lemma 5.2 to each of the quotients in (5.15).

Proof of Theorem 5.2. It follows from Def 3.2 of the "torsion index" $\boldsymbol{t}$ of $\boldsymbol{w}$ that when $\boldsymbol{s}>0$, there exists a Betti subgroup $\mathscr{B}$ of $A$ with a base $\left(u_{1}, \cdots, u_{r}\right)$ such that

$$
\boldsymbol{w}=\boldsymbol{s} u_{B}+\tau_{B} \quad\left(u_{B}=u_{1}, \text { order } \tau_{B}=\boldsymbol{t}\right) .
$$

If $\Psi: A \longrightarrow A / W$ is the natural homomorphism of $A$ onto $A / W$ it follows from Lemma 5.1 that

$$
\text { order } \left.\Psi\left(u_{B}\right)=\boldsymbol{s t} \quad \text { (when order } \tau_{B}=\boldsymbol{t}\right) \text {. }
$$

Нуротнеsis. We assume that when $\boldsymbol{s} \geq 1, \boldsymbol{w}$ is given a representation of form (5.16). This is a condition on $\tau_{B}$ and not on $\boldsymbol{w}$.

The following lemma is needed to establish the necessity of the conditions of Theorem 5.2 (i).

Lemma 5.3. If the free index $\boldsymbol{s}$ and torsion index $\boldsymbol{t}$ of $\boldsymbol{w}$ are relatively prime and $\boldsymbol{s}>0$, then $\boldsymbol{t}=1$.

Starting with (5.16) we note that

$$
\tau_{B} \in\left\{\tau_{B}\right\}=\left\{s \tau_{B}\right\} \subset s \mathscr{T} .
$$

The equality in (5.18) is valid since $s$ is prime to (order $\tau_{B}$ ). From (5.18) we infer the existence of an element $x \in \mathscr{T}$ such that $\tau_{B}=\boldsymbol{s} x$. We rewrite (5.16) in the form

$$
\boldsymbol{w}=\boldsymbol{s}\left(u_{B}+x\right)+\left(\tau_{B}-\boldsymbol{s} x\right)=\boldsymbol{s}\left(u_{B}+x\right) .
$$

From the given Betti group $\mathscr{B}$ one can obtain a second Betti group $\mathscr{B}^{\prime}$ of $A$ by replacing the generator $u_{B}$ of $\mathscr{B}$ by $u_{B}+x=u_{B \prime}$. In terms of $u_{B \prime}, \boldsymbol{w}=\boldsymbol{s} u_{B^{\prime}}$. This shows that $\boldsymbol{t}=1$.

Thus Lemma 5.3 is true. 
Proof of (ii) of Theorem 5.2. According to Lemma 3.4, when $s \geq 1$ the torsion subgroup $\hat{T}$ of $A / W$ satisfies the relation,

$$
\hat{T} \approx \frac{\left\{u_{1}\right\} \oplus \mathscr{T}}{W} . \quad(W=\{\boldsymbol{w}\})
$$

Since $\boldsymbol{t}=1$ by hypothesis of (ii), (5.16) gives $\boldsymbol{w}$ the form $\boldsymbol{w}=\boldsymbol{s} u_{B}=\boldsymbol{s} u_{1}$. Hence

$$
\hat{T} \approx \frac{\left\{u_{1}\right\} \oplus \mathscr{T}}{\left\{s u_{1}\right\} \oplus\{0\}} \approx C(s) \oplus \mathscr{T}
$$

where $C(\boldsymbol{s})$ is a cyclic group of order $\boldsymbol{s}$.

Statement (ii) of Theorem 5.2 follows from (5.21).

Necessity of the conditions of Theorem 5.2 (i). By Lemma 5.1, $\Psi\left(u_{B}\right)$ is an element of $A / W$ of order st. Hence if $A / W$ is a prime-simple group, st is a prime-simple number, that is, $\boldsymbol{s}$ must be prime-simple and relatively prime to $\boldsymbol{t}$. According to Lemma 5.3 it is accordingly necessary that $\boldsymbol{t}=1$.

Sufficiency of the conditions of Theorem 5.2 (i). The sufficiency follows from Theorem 5.2 (ii) and Theorem 1.4.

This completes the proof of Theorem 5.2.

\section{REFERENCES}

[1] Eilenberg, S. Singular homology theory, Ann. of Math., 45 (1944), 407-447.

[2] Morse, M. Topologically non-degenerate functions on a compact $n$-manifold $\mathrm{M}, J$. Analyse Math., 7 (1959), 189-208.

[ 3 ] Morse, M. and Cairns, S.S. Critical Point Theory in Global Analysis and Differential Topology. An Introduction. Academic Press, New York, 1969.

[4] Morse, M. Introduction to Analysis in the Large. 1947 Lectures. University Microfilms, Inc., Ann Arbor, Michigan, 1947.

[ 5 ] Morse, M. and Cairns, S.S. Singular homology over $\mathbf{Z}$ topological manifolds, J. Differential Geometry 3 (1969), $1 \sim 32$.

[6] Morse, M. The analysis and analysis situs of regular $n$-spreads in $(n+r)$-space, Proc. Natl. Acad. Sci. USA, 13 (1927), 813-817.

[ 7 ] Eells, J. and Kuiper, N.H. Manifolds which are like projective planes, Publ. Inst. Hautes Etudes Sci., 14 (1962), 181-222.

[8] Ledermann, W. Introduction to the Theory of Finite Groups. 5th ed. Interscience, New York, 1964.

[9] Ledermann, W. Introduction to the Theory of Finite Groups. 1st ed. Interscience, New York, 1949.

[10] Rotman, N.J. Theory of Groups: an Introduction. Allyn and Bacon, New York, 1965.

[11] Hall, M. The Theory of Groups. Macmillan, New York, 1959.

[12] Seifert, H. and Threlfall, W. Lehrbuch der Topologie. Teubner, Berlin, 1934. 
[13] Velben, O. Analysis Situs. 2nd ed. Amer. Math. Soc. Colloquium Publications, vol. 5, part II, Providence, R.I., 1960.

[14] Fuchs, L., Abelian Groups. Publishing House of the Hungarian Academy of Sciences, Budapest, 1958.

Institute for Advanced Study, Princeton, New Jersey University of Illinois, Urbana, Illinois 